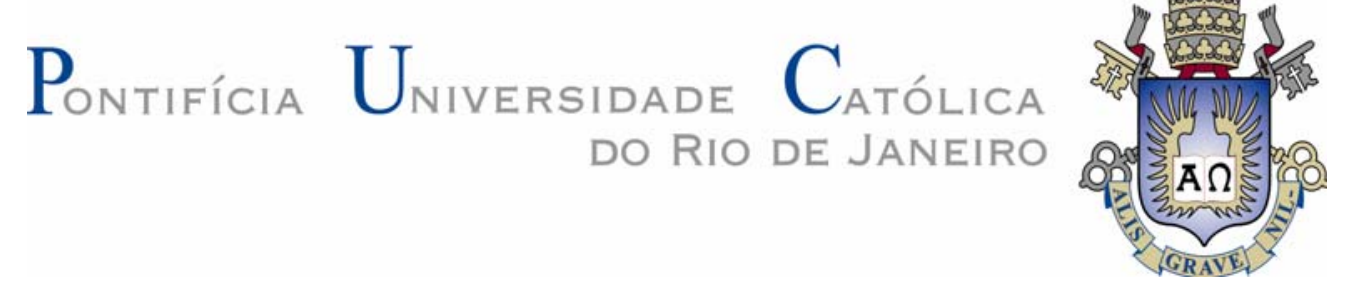

Marta Pudwell Chaves de Almeida

Modelamento de Efeitos de Propagação em Enlaces Satélite de Órbita Baixa

Tese apresentada como requisito parcial para obtenção do título de Doutor pelo Programa de PósGraduação em Engenharia Elétrica da PUC-Rio.

Orientador: Luiz Alencar Reis da Silva Mello

Rio de Janeiro

Agosto de 2003 


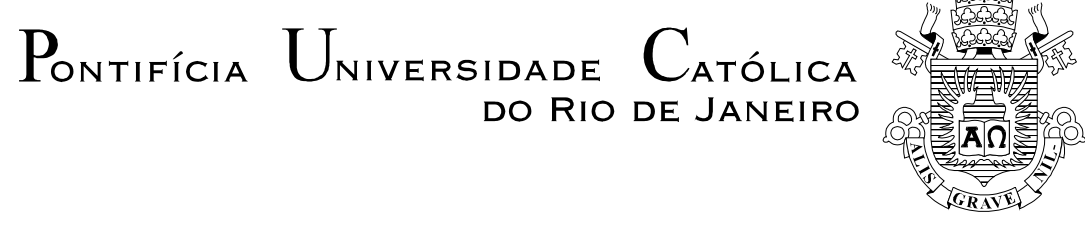

Marta Pudwell Chaves de Almeida

\title{
Modelamento de Efeitos de Propagação em Enlaces Satélite de Órbita Baixa
}

\begin{abstract}
Tese de Doutorado apresentada como requisito parcial para obtenção do grau de Doutor pelo Programa de PósGraduação em Engenharia Elétrica do Departamento de Engenharia Elétrica do Centro Técnico Científico da PUC-Rio. Aprovada pela Comissão Examinadora abaixo assinada..
\end{abstract}

Dr. Luiz Alencar Reis da Silva Mello Orientador Centro de Estudos de Telecomunicações - PUC-Rio

Dr. Erasmus Couto Brazil de Miranda Co-Orientador UCP

Dr. Luiz Cláudio Palma Pereira Fundação CPqD

Dr. Mauro Soares de Assis IME

Dr. Eduardo Javier Arancibia Vásquez TIM / Brasil

Dr. Marco Antonio Grivet Mattoso Maia Centro de Estudos de Telecomunicações - PUC-Rio

Prof. Ney Augusto Dumont Coordenador Setorial do Centro Técnico Científico - PUC-Rio 
Todos os direitos reservados. É proibida a reprodução total ou parcial do trabalho sem autorização da universidade, da autora e do orientador.

\section{Marta Pudwell Chaves de Almeida}

Graduou-se em Engenharia Elétrica com ênfase em Telecomunicações pela PUC-Rio em 1993. Fez Mestrado em Engenharia Elétrica, com área de concentração em Eletromagnetismo Aplicado, 1996.

Ficha Catalográfica

Almeida, Marta Pudwell Chaves de

Modelamento de efeitos de propagação em enlaces satélite de órbita baixa / Marta Pudwell Chaves de Almeida; orientador: Luiz Alencar Reis da Silva Mello. Rio de Janeiro: PUC, Departamento de Engenharia Elétrica, 2003.

141 f. :li. ; $30 \mathrm{~cm}$

Tese (doutorado) - Pontifícia Universidade Católica do Rio de Janeiro, Departamento de Engenharia Elétrica.

Inclui referências bibliográficas.

1. Engenharia elétrica - Teses. 2. Atenuação por chuvas. 3. Propagação de ondas radioelétricas. 4. Precipitação. 5. Enlaces satélite. 6. Sistemas de órbita baixa. 7. Satélites geoestacionários. I. Mello, Luiz Alencar Reis da Silva. II Pontifícia Universidade Católica do Rio de Janeiro. Departamento de Engenharia Elétrica. III. Título 


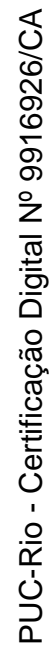

Ao meu marido

Alexandre

às nossas pequenas

Marianna e Manuela. 


\section{Agradecimentos}

Ao professor Silva Mello, meu orientador, por ter me guiado neste trabalho e por ter compreendido as mudanças em minha vida durante esta caminhada.

Ao amigo Erasmus, pelo incentivo e pela grande colaboração no desenvolvimento do trabalho.

Ao Rodolfo, pelo apoio na utilização do banco de dados, e ao Marcelo Jimenez pelo valioso auxílio durante o desenvolvimento do programa de simulação.

Ao CNPQ pelo apoio concedido para a realização do trabalho.

Ao Alexandre, meu marido dedicado, meu amigo e meu maior incentivador, pelo apoio incondicional que foi decisivo para a conclusão da tese.

Ao meu pai José, pelo estímulo contínuo, e muito especialmente à minha mãe Isolete, pelo carinho e pelo apoio diário.

À Rose, cuja função foi primordial para que eu pudesse me dedicar com tranquilidade a este trabalho. 


\section{Resumo}

Almeida, Marta Pudwell Chaves de. Modelamento de Efeitos de Propagação em Enlaces Satélite de Órbita Baixa. Rio de Janeiro, 2003. 141p. Tese de Doutorado - Departamento de Engenharia Elétrica, Pontifícia Universidade Católica do Rio de Janeiro.

O efeito da atenuação por chuvas em enlaces satélites que operam em frequências acima de $10 \mathrm{GHz}$ é decisivo no cálculo de desempenho do sistema. A evolução da tecnologia dos sistemas satélite e a necessidade de expansão dos serviços de comunicações móveis pessoais levaram ao uso de sistemas de comunicação com satélites de órbita baixa. Estes sistemas são particularmente interessantes para o oferecimento destes serviços por suas características de pequeno atraso de propagação e uso de potência de transmissão mais baixas que os sistemas geoestacionários. Satélites em órbita baixas (LEO) operam em altitudes de cerca de $1000 \mathrm{~km}$ e possuem movimento em relação à estação terrena. Esta característica de mobilidade traz novas questões sobre o comportamento da atenuação por chuvas, em particular a necessidade de um modelo de previsão de atenuação para estes enlaces com ângulo de elevação variante no tempo. Neste trabalho um modelo para a previsão de atenuação em enlaces com ângulo de elevação fixo, mais preciso do que os existentes na literatura técnica, foi desenvolvido como primeiro passo para o tratamento do problema de enlaces com ângulo de elevação variável. A seguir foi feita uma simulação de medidas em enlaces LEO a partir do banco de dados de medidas de atenuação em enlaces fixos no Brasil, considerando um modelo de constelação de satélites. Foi implementado um método geral que utiliza o histograma dos ângulos de elevação do satélite como ponderação da distribuição cumulativa de atenuação em cada ângulo. $O$ método foi testado contra os resultados obtidos com simulações para a constelação Globalstar sendo obtida muito boa concordância entre o modelo de predição desenvolvido e a simulação.

\section{Palavras-chave}

Atenuação por chuvas, propagação de ondas radioelétricas, precipitação, enlaces satélite, sistemas de órbita baixa, satélites geoestacionários. 


\begin{abstract}
Almeida, Marta Pudwell Chaves de. Modelamento de Efeitos de Propagação em Enlaces Satélite de Órbita Baixa. Rio de Janeiro, 2003. 141p. PhD Thesis - Electrical Engineering Department, Pontifícia Universidade Católica do Rio de Janeiro.
\end{abstract}

Rain attenuation is the most important propagation effect to be taken into account in the performance calculation for satellite systems operation at frequencies above $10 \mathrm{GHz}$. The technological evolution of such systems and the need for personal communication systems with global coverage lead to the use of low earth orbit communication systems, that not only have shorter propagation delays but also allow the use of lower transmission power than the traditional geostationary systems. Low earth orbit (LEO) satellites have altitudes around $1.000 \mathrm{Km}$ and are in motion relatively to the earth stations. This mobility requires a new approach to the problem of rain attenuation prediction, particularly the need for a prediction model that takes into account the elevation angle variability. In this work, an improved rain attenuation prediction method for the geostationary case has been developed as a starting point for the analysis of the nongeostationary case. Then, the rain attenuation in the a LEO system has been simulated using measured data from fixed system systems and the satellite constellation model. A general method for slant path rain attenuation prediction considering variable elevation angles is proposed. The method uses the histogram of the elevation angles to weight the distributions obtained for fixed elevation. The method has been tested with simulations performed for the Globalstar LEO system and a very good agreement was obtained.

\title{
Key words
}

Satellite links, rain attenuation, radio propagation. 


\section{Sumário}

1 Introdução 15

1.1. Sistemas Satélite Não Geoestacionários 19

1.2. Alocação de Freqüências 22

1.3. Sistema de Rastreamento de Satélites 22

1.4. Atenuação por Chuvas 24

1.4.1. Atenuação específica 24

1.4.2. Modelagem da estrutura da chuva 25

1.4.3. Modelos de previsão de atenuação da UIT-R e CETUC 27

2 Modelagem da previsão de atenuação por chuvas em enlaces GEO 34

2.1. Preparação do Banco de Dados 36

2.2. Métodos utilizados na estimação dos parâmetros de $L_{o}$ e $h_{r} \quad 36$

2.3. Procedimento de modelagem dos parâmetros de $L_{o}$ e $h_{r} \quad 37$

2.4. Aplicação dos modelos de previsão Cetuc/BR e Cetuc/Global no Brasil 41

2.5. Comparação entre o modelo desenvolvido e o modelo UIT-R 43

$\begin{array}{ll}\text { 2.6. Conclusão } & 46\end{array}$

3 Desenvolvimento da fórmula de conversão de atenuação 48

3.1. Banco de dados de atenuação e taxa de precipitação 49

3.2. Determinação da fórmula de conversão de atenuação 50

3.3. Validação da fórmula de conversão - Tipo I 53

3.3.1. Resultados da aplicação das conversões nas distribuições $\begin{array}{ll}\text { cumulativas de atenuação } & 54\end{array}$

3.3.2. Comparação entre os modelos de conversão do Tipo I 57

3.4. Validação das fórmulas de conversão - Tipo II 58

3.4.1. Aplicação da conversão na série temporal de atenuação 58

3.4.2. Elaboração do programa de cálculo 59

3.4.3. Descrição do Programa\#1 - cálculo de distribuições cumulativas 60 
3.4.4. Resultados da validação com conversão direta na série temporal de $\begin{array}{ll}\text { atenuação } & 61\end{array}$

3.4.5. Comparação dos modelos de conversão do Tipo II 64

3.5. Conclusões 65

4 Caracterização do ângulo de elevação variante no tempo 66

4.1. Implementação do modelo orbital 68

4.2. Modelo orbital com coordenadas cartesianas 69

4.3. Elementos Orbitais Keplerianos 76

4.3.1. Nomenclatura dos elementos orbitais $\quad 76$

$\begin{array}{ll}\text { 4.4. Perturbações Orbitais } & 78\end{array}$

4.5. Procedimento do cálculo de elevação variante no tempo 82

4.5.1. Constelação de satélites LEO 82

4.5.2. Banco de Dados do movimento da constelação 83

4.5.3. Estatísticas do ângulo de elevação 88

5 Modelagem da previsão de atenuação por chuvas em enlaces LEO 104

5.1. Modelo de previsão de atenuação em enlaces NGEO proposto pela

$\begin{array}{ll}\text { UIT-R } & 105\end{array}$

5.2. Simulação das medidas de atenuação dinâmica 106

5.3. Método de previsão de atenuação baseado no modelo UIT-R 112

5.4. Aplicação do modelo de distribuição do ângulo de elevação 113

5.5. Comparação dos resultados 113

6 Conclusões 118

$\begin{array}{ll}\text { 6.1. Sugestões de trabalhos futuros } & 120\end{array}$ 


\section{Lista de figuras}

Figura 1 - Célula de chuva equivalente 26

Figura 2 - Geometria do deslocamento da célula de chuva 28

Figura 3 - Posições relativas da cél. em relação ao enlace no caso $L_{0} \geq \operatorname{Lscos} \theta 29$

Figura 4-Geometria da primeira integral 29

Figura 5-Geometria da segunda integral 29

Figura 6-Geometria da terceira integral $\quad 30$

Figura 8 - Plotagem dos resíduos do Modelo Cetuc/BR segundo valores da distribuição normal 38

Figura 9 - Valores previstos versus observados $\quad 38$

Figura 10 - Distribuição dos resíduos do Modelo Cetuc/Global $\quad 40$

Figura 11 - Valores previstos versus observados $\quad 41$

Figura 12 - Previsão de atenuação por dois modelos em Mosqueiro (PA) 42

Figura 13 - Previsão de atenuação por dois modelos no Rio de Janeiro (RJ), período 1

Figura 14 - Previsão de atenuação por dois modelos no Rio de Janeiro (RJ), período 2

Figura 15 - Previsão de atenuação por dois modelos em Curitiba (PR), período 1

Figura 16 - Previsão de atenuação por dois modelos em Porto Alegre (RS) 43

Figura 17 - Erro relativo médio $\quad 44$

Figura 18 - Desvio padrão $\quad 45$

Figura 19 - Erro RMS $\quad 45$

Figura 20 - Valor de Vi médio 45

Figura 21 - Desvio padrão de Vi 46

Figura 22 - Valor RMS de Vi 46

Figura 23 - Distribuições cumulativas medidas e convertida $53^{\circ}-90^{\circ}$, período 255

Figura 24 - Conversão $53^{\circ}-90^{\circ}$ pelo modelo Cetuc, período 2

Figura 25 - Distribuições cumulativas medidas e convertida $90^{\circ}-53^{\circ}$, período 256

Figura 26 - Conversão $90^{\circ}-53^{\circ}$, período 2 
Figura 27 - Estrutura geral do Programa \#1

Figura 28 - Gráfico das séries de atenuação RA medida, convertida para $90^{\circ} \mathrm{e}$ chuva

Figura 29 - Distrib. cumulativas medidas e convertidas $53^{\circ}-90^{\circ}$, período $2 \quad 62$

Figura 30 - Conversão $53^{\circ}-90^{\circ}$, período 2

Figura 31 - Distrib. cumulativas medidas e convertidas $90^{\circ}-53^{\circ}$, período 263

Figura 32 - Conversão $90^{\circ}-53^{\circ}$, período 2

Figura 33 - Coordenadas do plano orbital $\mathrm{x}_{0}, \mathrm{y}_{0}, \mathrm{z}_{0}$ e coordenadas ECI x,y,z 70

Figura 34 - Sistema de coordenadas inerciais centradas na Terra (ECI) 71

Figura 35 - Geometria do meridiano de Greenwich e do meridiano do ponto subsatélite $\quad 72$

Figura 36 - Coordenadas ECF x', $\mathrm{y}^{\prime}, \mathrm{z}^{\prime}$ e coordenadas topocêntricas $\mathrm{x}_{\mathrm{t}}, \mathrm{y}_{\mathrm{t}}, \mathrm{z}_{\mathrm{t}} \quad 75$

Figura 37 - Geometria da Terra oblata $\quad 80$

Figura 38 - Variação do ângulo de elevação de dois satélites, visto do Rio de Janeiro

Figura 39 - Intervalo de visibilidade de dois satélites $\quad 84$

Figura 40 - Estrutura geral dos Programas \#2 - Gerador de Órbita 85

Figura 41 - Envoltória do ângulo de elevação visto de Mosqueiro 85

Figura 42 - Envoltória do ângulo de elevação visto de Recife 86

Figura 43 - Envoltória do ângulo de elevação visto do Rio de Janeiro 86

Figura 44 - Envoltória do ângulo de elevação visto de Curitiba 86

Figura 45 - Envoltória do ângulo de elevação visto de Porto Alegre 87

Figura 46 - Envoltória em intervalo de 2 horas 87

Figura 47 - Histograma médio de Mosqueiro $\quad 89$

Figura 48 - Histograma médio de Recife $\quad 89$

Figura 49 - Histograma médio do Rio de Janeiro $\quad 89$

Figura 50 - Histograma médio de Curitiba 90

Figura 51 - Histograma médio de Porto Alegre 90

$\begin{array}{ll}\text { Figura } 52 \text { - Variação dos histogramas com a latitude } & 91\end{array}$

Figura 53 - Correlação entre histogramas da latitude $-40^{\circ} 92$

Figura 55 - Histogramas em latitudes simétricas na longitude zero 93

Figura 57 - Variação do fator de escala com a latitude 96

Figura 58 - Destaque da posição do modo na latitude $45^{\circ}$ 
Figura 59 - Ajuste do fator de escala na faixa $0^{\circ}$ a $40^{\circ} \quad 97$

Figura 60 - Ajuste do fator de escala na faixa de $50^{\circ}$ a $70^{\circ} \quad 97$

Figura 61 - Variação do fator de forma com a latitude 98

Figura 62 - Ajuste do fator de forma na faixa de $0^{\circ}$ a $40^{\circ} 98$

Figura 63 - Ajuste do fator de forma na faixa de $45^{\circ}$ a $70^{\circ} \quad 99$

Figura 64 - Variação do fator de deslocamento com a latitude 99

Figura 65 - Ajuste do fator de deslocamento na faixa de $0^{\circ}$ a $40^{\circ} 99$

Figura 66 - Ajuste do fator de deslocamento na faixa de $45^{\circ}$ a $70^{\circ} \quad 100$

Figura 67 - Comparação entre as distrib. estimada e ajustada pelo modelo 101

Figura 68 - Histogramas medido e calculado para Mosqueiro 102

Figura 69 - Histogramas medido e calculado para Recife 102

Figura 70 - Histogramas medido e calculado para o Rio de Janeiro 103

Figura 71 - Histogramas medido e calculado para Curitiba 103

Figura 72 - Histogramas medido e calculado para Porto Alegre 103

Figura 73 - Eventos de atenuação e chuva 107

Figura 74 - Fatores de conversão para Mosqueiro (PA) 108

Figura 75 - Fatores de conversão para o Rio de Janeiro (RJ) 108

Figura 77 - Simulação de medidas dinâmicas em Mosqueiro 109

Figura 78 - Simulação de medidas dinâmicas em Recife, período 1

Figura 79 - Simulação de medidas dinâmicas em Recife, período $2 \quad 110$

Figura 80 - Simulação de medidas dinâmicas no Rio de Janeiro, período 1110

Figura 81 - Simulação de medidas dinâmicas no Rio de Janeiro, período 2110

Figura 82 - Simulação de medidas dinâmicas em Curitiba 111

Figura 83 - Simulação de medidas dinâmicas em Porto Alegre. 111

$\begin{array}{ll}\text { Figura } 84 \text { - Resultados para Mosqueiro } & 114\end{array}$

Figura 85 - Resultados para Recife, período 1

Figura 86 - Resultados para Recife, período $2 \quad 114$

Figura 87 - Resultados para Rio de Janeiro, período 1

Figura 88 - Resultados para Rio de Janeiro, período 2

Figura 89 - Resultados para Curitiba $\quad 115$

Figura 90 - Resultados para Porto Alegre 116 


\section{Lista de tabelas}

Tabela 1 - Valores dos parâmetros da Modelagem Cetuc/BR 37

Tabela 2 - Parâmetros para o Modelo Cetuc/Global 40

Tabela 3 - Locais e tipos de medidas $\quad 49$

Tabela 4 - Distribuições cumulativas de atenuação e chuvas medidas no Rio de Janeiro nos períodos 1 e 2

Tabela 5 - Distribuições cumulativas para período $2 \quad 57$

Tabela 6 - Erro médio, desvio padrão e valor RMS para o período 1

Tabela 7 - Erro médio, desvio padrão e valor RMS para o período $2 \quad 58$

Tabela 8 - Distribuições cumulativas para o período 2, conversão Tipo II 64

Tabela 9 - Erro médio, desvio padrão e valor RMS para o período 1, conversão Tipo II 64

Tabela 10 - Erro médio, desvio padrão e valor RMS para o período 2, conversão Tipo II 64

Tabela 11 - Valores do TLE $\quad 78$

Tabela 12 - Características dos locais de medidas $\quad 85$

Tabela 13 - Parâmetros de Weibull para cada latitude 96

Tabela 14 - Características de cada local de medida e faixa de valores de fator de $\begin{array}{ll}\text { conversão } & 108\end{array}$

Tabela 15 - Erro, desvio padrão e valor RMS para Mosqueiro 116

Tabela 16 - Erro, desvio padrão e valor RMS para Recife período I 117

Tabela 17 - Erro, desvio padrão e valor RMS para Recife período II 117

Tabela 18 - Erro, desvio padrão e valor RMS para o Rio de Janeiro período I 117

Tabela 19 - Erro, desvio padrão e valor RMS para o Rio de Janeiro período II 117

Tabela 20 - Erro, desvio padrão e valor RMS para Curitiba 117

Tabela 21 - Erro, desvio padrão e valor RMS para Porto Alegre 117 


\section{GLOSSÁRIO}

$\begin{array}{ll}\text { ITU } & \text { International Telecommunications Union } \\ \text { UIT } & \text { União Internacional das Telecomunicações } \\ \text { LEO } & \text { Low Earth Orbit } \\ \text { MEO } & \text { Medium Earth Orbit } \\ \text { HEO } & \text { High Elliptical Orbit } \\ \text { GEO } & \text { Geostationary Orbit } \\ \text { NGEO } & \text { Nongeostationary Orbit } \\ \text { MSS } & \text { Mobile Satellite Service } \\ \text { FSS } & \text { Fixed Satellite Service } \\ \text { WGS 84 } & \text { World Geodetic System do ano } 1984 \\ \text { ECI } & \text { Earth Centered Inertial } \\ \text { ECF } & \text { Earth Centered Fixed }\end{array}$

